\title{
Programa de prevención y control de las enfermedades respiratorias agudas de la infancia en Santiago, Chile
}

\author{
Leonardo Véjar M., ${ }^{1}$ Carlos Castillo D., ${ }^{1}$ Patricia Navarrete M. ${ }^{1}$ \\ y Salomé Sánchez C. ${ }^{1}$
}

RESUMEN En el presente trabajo se describen y examinan las actividades de un programa pediátrico para la prevención y el control de las infecciones respiratorias agudas (IRA) en un servicio de salud estatal que atiende a la población de bajos ingresos de Santiago, Chile. En un área de salud correspondiente a una población de algo más de un millón de habitantes, con un hospital de base, 16 consultorios de atención primaria y ocho postas rurales, se desarrollaron actividades específicas de capacitación de personal, así como acciones de control en atención primaria y de hospitalización de niños con IRA grave. En los primeros 5 años de funcionamiento del programa (1990-1994) se observó entre niños menores de un año una disminución de la mortalidad anual por neumonía de 3,0 a 1,7 por 1 000, lo que ha contribuido a un descenso de la mortalidad infantil de 13,5 a 8,9 defunciones por 1000 nacidos vivos. En 1994 los costos del programa alcanzaron los US\$224 000. A juzgar por los resultados obtenidos, el programa de prevención y control de IRA puede haber sido un aporte valioso a la salud infantil del área.

La infección respiratoria aguda (IRA) constituye una de las causas principales de morbilidad y mortalidad en la infancia y cada año es responsable del fallecimiento de cuatro millones de niños en todo el mundo (1). La meta propuesta por la OMS en 1990 fue conseguir que para el año 2000 las muertes por neumonía se hayan reducido a un tercio de su número actual (2).

En Chile, la disminución durante las últimas décadas de las defunciones infantiles por enfermedades diarreicas

\footnotetext{
1 Hospital Dr. Sótero del Río, Servicio de Pediatría, Santiago, Chile. Toda correspondencia relacionada con este artículo debe enviarse al primer autor a la siguiente dirección postal: Vicuña Mackenna 6770, La Florida, Santiago, Chile.
}

e infectocontagiosas y por trastornos asociados con la desnutrición ha convertido a la neumonía en la causa más importante de mortalidad evitable en la niñez (3-5). El área de la ciudad de Santiago atendida por el Servicio de Salud Metropolitano Suroriente, que es uno de los 26 servicios de salud estatales del país, ha sido representativa de esa evolución, con ciertas características especiales. Por ser un área geográfica muy grande con extensas zonas agrícolas hasta hace apenas un decenio, recibió una enorme emigración tanto de otras áreas del país como de otras partes de Santiago, de tal forma que en 10 años la población que atiende el servicio se duplicó hasta llegar a ser de algo más de un millón de habitantes en 1994. Actual- mente, una gran proporción de esta población vive en condiciones de pobreza o extrema pobreza. La atención de salud la proporciona una red de 16 consultorios urbanos, ocho postas rurales y un hospital de referencia con 0,3 camas por cada 1000 menores de 15 años (6).

La mortalidad infantil en la zona está en los promedios nacionales y la mortalidad posneonatal no llega a ser $50 \%$ de la infantil. La mayor parte de las defunciones por neumonía que se produjeron en 1990 tuvieron lugar en los hogares y según los datos de autopsia las neumonías fueron en la mayor parte de los casos de origen viral $(7,8)$.

Siguiendo los planteamientos de la OPS/OMS $(2,9)$, que fueron impulsa- 
dos a su vez por el Ministerio de Salud de Chile, a fines de 1989 se inició en la zona un programa de control de las IRA infantiles. Este programa se articuló en el nivel nacional con un programa de control de la obstrucción bronquial organizado por el Ministerio de Salud (10) y aplicado en el ámbito de la atención primaria. Las metas eran reducir la mortalidad por neumonía en menores de 5 años y en especial en menores de 1 año, reducir la mortalidad domiciliaria por neumonía, aumentar las hospitalizaciones de niños con IRA grave y el tratamiento en consultorio de los niños con síndrome bronquial obstructivo, y mejorar el tratamiento en el hogar de las IRA menos graves. Este programa se ha desarrollado mediante diversas estrategias de educación y aplicación de esquemas terapéuticos específicos.

El presente trabajo describe los componentes principales del programa y muestra sus resultados en los primeros 5 años de funcionamiento. También examina el impacto que ha tenido el programa en los indicadores de salud infantil.

\section{EVALUACIÓN DEL PROGRAMA}

Se analizaron las actividades del Programa de Prevención y Control de IRA en los 16 consultorios de atención primaria y en las ocho postas rurales del Servicio de Salud Metropolitano Suroriente, y se evaluaron sus efectos sobre los indicadores de morbilidad y mortalidad posneonatal en el período inicial de aplicación del programa entre 1990 y 1994. También se analizaron las actividades relacionadas con las IRA en los niveles secundario y terciario de la atención sanitaria del área en cuestión.

Se revisaron los registros de actividades del Programa de IRA en el Servicio de Salud Metropolitano Suroriente de Santiago, los registros de hospitalizaciones por IRA en el servicio de pediatría del Hospital Dr. Sótero del Río y el registro computadorizado de las hospitalizaciones del servicio de pediatría. El número de de- funciones por neumonía se corroboró con el indicado en el registro de mortalidad infantil del Ministerio de Salud y con las cifras del Instituto Nacional de Estadísticas (11). Las defunciones en el domicilio se analizaron según los datos de los registros locales del Programa de IRA. Los certificados de defunción correspondientes a estos casos se hacen casi siempre en el servicio de anatomía patológica del hospital, ya que por norma local todas las muertes en un domicilio deben someterse a autopsia y a examen anatomopatológico con microscopio (8).

\section{DESARROLLO DEL PROGRAMA}

El programa se inició con reuniones informativas con las autoridades de salud del área y las jefaturas de los municipios y se extendió a los consultorios de atención primaria mediante actividades de capacitación sobre IRA. En cada consultorio se organizó un ciclo de talleres con una duración individual promedio de 16 horas y orientados a todo el personal de salud, que incluía médicos, enfermeras, otros profesionales y personal auxiliar. Los contenidos docentes corrieron a cargo de un grupo de especialistas en medicina broncopulmonar, pediatras y médicos generales. Durante la capacitación se hizo hincapié en los signos sugerentes de gravedad y en los conceptos de atención focalizada de grupos de riesgo y de acceso al sistema de salud.

Los recursos humanos, que consistieron inicialmente en tres médicos generales con dedicación de media jornada y cinco quinesiólogos, llegaron a ser finalmente cinco médicos generales y 17 quinesiólogos (o sea, uno por cada consultorio de atención primaria, con dedicación de 6 horas diarias). Usualmente este equipo se completaba con un médico y una auxiliar o enfermera asignada de los recursos propios del consultorio.

Los equipos eran coordinados centralmente por un comité formado por una enfermera, un representante de la dirección del servicio de salud, el jefe de la unidad broncopulmonar del hospital (jefe del programa de IRA) y un educador sanitario.

En cada consultorio se contaba con una habitación denominada sala de hospitalización abreviada $(12,13)$, con camilla, oxígeno, máquina de aspiración-nebulización, broncodilatadores en aerosol y registros.

Según la normativa del programa, el equipo de IRA de cada consultorio tenía a su cargo la selección de los pacientes más críticos; el tratamiento de las IRA menos graves, incluido el tratamiento de los pacientes con síndrome de obstrucción bronquial en la sala de hospitalización abreviada; el envío de pacientes con IRA grave al hospital, según las normas modificadas de la OPS, y la educación de las familias de pacientes en grupos de riesgo, así como de la comunidad y del resto del equipo de salud del consultorio. Para ello se disponía de videos, manuales y trípticos editados localmente.

En la hospitalización abreviada se aplica a todos los niños con síndrome bronquial obstructivo un esquema estandarizado para diagnosticar el grado de obstrucción bronquial y se obtiene la regresión del síndrome mediante broncodilatadores en aerosol a presión del tipo MDI (13). Los que no mejoran se consideran casos de neumonía probables y se envían al hospital. Entre 1990 y 1994 se practicaron, respectivamente, 378, 410, 540 y 444 hospitalizaciones abreviadas.

El número de egresos del servicio de pediatría del Hospital Dr. Sótero del Río aumentó de 2756 en 1990 a 4068 en 1994, el promedio de días de hospitalización disminuyó de 7,9 a 6,3 y la letalidad descendió de 2,7 a 1,3\%. El hospital no disponía de información desagregada por diagnósticos, pero más de $70 \%$ de los egresos del servicio de pediatría correspondieron a neumonías que ocurrieron en los meses fríos del año.

Se observó en el área del servicio de salud una disminución progresiva de la mortalidad general por IRA y de los niños fallecidos por la misma causa en su domicilio (cuadro 1). La mortalidad por neumonía en menores de 5 años 
CUADRO 1. Mortalidad infantil y mortalidad por infecciones respiratorias agudas en niños menores de 5 años. Servicio de Salud Metropolitano Suroriente, Santiago, Chile, 1990-1994

\begin{tabular}{|c|c|c|c|c|c|}
\hline \multirow[b]{2}{*}{ Indicador } & \multicolumn{5}{|c|}{ Año } \\
\hline & 1990 & 1991 & 1992 & 1993 & 1994 \\
\hline Defunciones por IRA en menores de 5 años & 80 & 79 & 78 & 55 & 50 \\
\hline Defunciones por IRA en menores de 1 año & 73 & 75 & 72 & 49 & 43 \\
\hline $\begin{array}{l}\text { Defunciones por IRA en menores de } 5 \text { años en su } \\
\text { domicilio }\end{array}$ & - & 59 & 48 & 37 & 33 \\
\hline $\begin{array}{l}\text { Tasa de mortalidad por IRA (por } 100000 \text { menores } \\
\text { de } 5 \text { años) }\end{array}$ & 69,4 & 67,5 & 64,2 & 47,0 & 37,7 \\
\hline $\begin{array}{l}\text { Tasa de mortalidad por IRA en menores de } 1 \text { año } \\
\text { (por } 1000 \text { nacidos vivos) }\end{array}$ & 3,2 & 3,2 & 2,9 & 1,9 & 1,7 \\
\hline $\begin{array}{l}\text { Tasa de mortalidad infantil (defunciones de menores } \\
\text { de un año por } 1000 \text { nacidos vivos) }\end{array}$ & 13,5 & 12,9 & 11,2 & 10,8 & 8,9 \\
\hline $\begin{array}{l}\text { Tasa de mortalidad neonatal (defunciones de } \\
\text { menores de un mes por } 1000 \text { nacidos vivos) }\end{array}$ & 5,9 & 6,5 & 6,1 & 6,1 & 4,8 \\
\hline
\end{tabular}

disminuyó de 69,4 a 37,7 por 100000 . Las defunciones domiciliarias bajaron de 59 en 1991 a 33 en 1994, o sea, de 50,4 a 24,9 por 100000 niños. El número de menores de un año que murieron de IRA descendió de 73 a 43, lo que corresponde a una reducción de la tasa de 3,2 a 1,7 por 1000 .

Los costos anuales del programa en el servicio de salud analizado han sido los siguientes, en pesos chilenos y dólares: \$ 6562900 (US\$ 21417 ) en 1990; \$ 20530025 (US\$ 58 508) en 1991; $\$ 44956407$ (US\$123 833) en 1992; $\$ 57136068$ (US\$140591) en 1993 y $\$ 94008009$ (US\$ 223 834) en 1994.

\section{DISCUSIÓN}

En Chile, las enfermedades respiratorias agudas del lactante son causadas principalmente por virus y en menor proporción por bacterias $(8$, 14-18). El estado de la inmunidad celular y humoral en los primeros meses de la vida, los requerimientos de oxígeno relativamente altos, la inmadurez de las vías respiratorias y la mayor fatigabilidad de la musculatura respiratoria $(19,20)$ hacen que el lactante sea muy susceptible a infecciones respiratorias que pueden evolucionar hacia la gravedad en poco tiempo, dando lugar a una obstrucción bronquial o bronconeumonía.
La fisiopatología de las IRA ha llevado a proponer métodos abreviados de muy alta sensibilidad mediante los cuales las madres pueden detectar los casos más graves que requieren de una evaluación por los equipos de salud. Los más destacados son la respiración rápida y los signos de dificultad respiratoria $(8,21-23)$. En el Servicio de Salud Metropolitano Suroriente la aplicación de estas estrategias coincidió con una reducción de la mortalidad por IRA. Esta disminución se dio al mismo tiempo que la población atendida por el servicio aumentó en 15\% (de 115000 menores de 5 años en 1990 a 132000 en 1994), aumento apreciable fundamentalmente entre los grupos de bajos recursos económicos.

La efectividad del programa está demostrada por el descenso de la mortalidad por IRA y sobre todo por la disminución de las defunciones en el domicilio. Estas últimas, que se asocian con condiciones de extrema pobreza, desnutrición y bajo peso al nacer $(8,24-25)$, disminuyeron a pesar de no haberse modificado significativamente las condiciones básicas de los hogares y de los niños. El programa se centró en promover la consulta, dar atención prioritaria al grupo de riesgo y tener disponibles recursos hospitalarios para ingresar a los niños (7).

Es posible que factores tales como las mejorías en la vivienda, en el sa- neamiento ambiental o, en general, en el nivel socioeconómico hayan influido directa o indirectamente en el descenso de la mortalidad infantil por IRA. No tenemos elementos para descartar su participación o confirmar su impacto. Puede que los servicios de atención de urgencia que se organizaron en algunos consultorios también hayan tenido un efecto. No obstante, al compararse las tendencias observadas en nuestro servicio con las advertidas en otros servicios de salud de condiciones similares en que el Programa de IRA no se implementó o se implementó solamente en la atención terciaria, se comprueba que la disminución de la mortalidad fue mayor en el nuestro (11).

Es probable que las estrategias más beneficiosas del programa fueran la capacitación y la motivación permanentes del personal de los equipos de salud. Alrededor de $50 \%$ del personal de los consultorios, o cerca de $90 \%$ del personal directamente responsable del manejo de niños menores de 5 años (unas 900 personas en total, entre personal auxiliar y profesional), participó en las actividades educativas.

Las actividades de educación dirigidas a las madres de niños en riesgo tuvieron un efecto más difícil de ponderar. Anualmente hay más de 16000 madres en el área cuyos hijos menores de un año se encuentran en riesgo y en el mejor año se estableció contacto con 3990 de ellas. La educación de la comunidad también incluyó la formación de voluntarios en el monitoreo de IRA. Sin embargo, no hay registro de su actividad ni posibilidad, por ahora, de evaluar su impacto relativo dentro del programa.

El desarrollo de salas de hospitalización abreviada en los consultorios puede haber tenido un efecto significativo en el manejo precoz de las crisis bronquiales obstructivas, con la consiguiente prevención del agravamiento y de la necesidad de hospitalización $(12,13)$. Sin embargo, es difícil evaluar cuán eficazmente se previnieron los ingresos. Por la escasez de cunas, la unidad de emergencia pediátrica del hospital había tenido que manejar 
localmente casos de obstrucción bronquial desde antes del desarrollo del programa. Esta situación con frecuencia impuso una sobrecarga a su capacidad resolutiva.

El aumento de camas hospitalarias y de los recursos humanos asociados (auxiliares de enfermería y médicos) destinados específicamente a la atención de IRA parece haber incidido muy favorablemente sobre las cifras correspondientes de mortalidad. Los egresos totales del servicio de pediatría aumentaron y hubo un descenso simultáneo del promedio de días de hospitalización y de la letalidad intrahospitalaria en general. No se dispone de información desagregada sobre el incremento de los casos que egresan con diagnóstico de IRA, pero la capacidad del servicio para resolver casos de IRA mejoró. En la disminución de la letalidad pueden haber tenido una influencia importante los cambios de la estructura del servicio, en particular la creación de salas de cuidados pro- gresivos y la consiguiente mejoría del manejo de casos graves.

La implantación del programa en los meses de invierno se vio dificultada por la falta de personal idóneo dispuesto a trabajar solamente 3 ó 4 meses al año.

Cabe preguntarse hasta cuándo será necesario prolongar el programa, sobre todo ante el hecho de que las cifras de mortalidad muestran la tendencia a estabilizarse. A juzgar por lo sucedido con otros programas (9), como los de desnutrición infantil, llegará una fase en el desarrollo nacional en que las condiciones socioeconómicas de los beneficiarios se alterarán y permitirán restringir o focalizar el programa en grupos de alto riesgo.

El éxito aparente del programa de IRA obliga a mejorar los sistemas para evaluar su impacto general y el de sus componentes específicos. A este objetivo debería contribuir un trabajo más integrado entre los diversos niveles de atención y la administración de salud municipalizada que se encarga de la atención primaria y de los servicios de salud.

En vista de los elevados costos del programa, se torna necesario determinar cuán indispensables son todas sus partes y si es posible adoptar medidas preventivas. También destaca la buena colaboración entre los grupos técnicos que lo han llevado a cabo (el servicio de salud) y el nivel político (el Ministerio de Salud) que ha decidido, en el marco de sus prioridades, asignar recursos para ese fin.

Agradecimiento. Agradecemos a Laboratorio Chile, S.A., la cooperación que nos brindó durante 3 años en diversas actividades del programa. Se agradece también a Marcela Vargas Leal su colaboración en las actividades de secretariado y preparación del manuscrito.

\section{REFERENCIAS}

1. Pio A. Acute respiratory infections in children in developing countries: an international point of view. Pediatr Infect Dis 1986;5:179-185.

2. Organización Panamericana de la Salud. Informe Programa IRA de la OMS. Washington, DC: OPS; 1990.

3. Toro JA. Muertes por neumonía: prioridad en control. Rev Enf Resp Cir Torac 1977;3:222-224.

4. Instituto Nacional de Epidemiología "Emilio Coni". Infecciones respiratorias agudas en los niños de los países de América: "Mortalidad en el Cono Sur". OPS/OMS: Santa Fe, Argentina; 1989.

5. Organización Panamericana de la Salud. Infecciones respiratorias agudas en las Américas. Washington, DC: OPS; 148. (Serie Paltex PXE25).

6. Secretaría Regional Ministerial de Salud. Anuario estadístico. Santiago, Chile: SRMS; 1992.

7. Véjar L. Manual para atención primaria de salud: infecciones respiratorias y tuberculosis en la infancia. Santiago, Chile: Ministerio de SaludSSMSO; 1992.

8. Casterán J, Carrasco R, Luco L, López I. Muertes infantiles extrahospitalarias. Rev Chil Pediatr 1991;62:390-395.

9. Organización Mundial de la Salud. La Asamblea Mundial de la Salud aprueba el Séptimo Programa General de Trabajo. Crónica de la OMS 1982;336:139-161.
10. Abara S, Astudillo P, Girardi G. Manejo del síndrome bronquial obstructivo en sala de prehospitalización. Rev Chil Enf Resp 1990;6: 192-197.

11. Vargas N. Mortalidad infantil: Chile 1994. Rev Chil Pediatr 1996;67:8.

12. Willert C, Davis T, Hernan J, et al. Short term holding room treatment of asthmatic children. J Pediatr 1985;5:707-713.

13. Astudillo P, Mancilla P, Girardi G, Aranda C. Hospitalización abreviada en 3268 lactantes con SBO. Enf Resp Cir Torac 1990;6:192-197.

14. Gallo A, Puentes R, Kunstmann S, Arenas E, Riedel I. Etiología de las neumopatías agudas del lactante. Rev Chil Pediatr 1982;53:570-576.

15. Vicente M, Wu E, Carrasco L, et al. Participación viral en las infecciones respiratorias agudas bajas del lactante. Rev Chil Pediatr 1988; 59:353-357.

16. Papic Z, Rodríguez L, Avendaño L. Virus respiratorios en lactantes con infecciones respiratorias altas y bajas. Rev Chil Pediatr 1992;63: 256-261.

17. Palomino M. Infecciones intra y extrahospitalarias por virus sincitial respiratorio en lactantes. Rev Chil Pediatr 1994;65:11-16.

18. Ceruti E, Díaz A, Vicente M, Escobar A, Martínez F, Pinto R, et al. Etiología de las IR bajas agudas en lactantes hospitalizados. Rev Chil Pediatr 1991;62:155-156.
19. Hawley ME, Bone RC. Acute respiratory failure: pathophysiology, causes and clinical manifestations. Postgrad Med 1986;79:166-178.

20. Nichols DG. Respiratory muscle performance in infants and children. I Pediatr 1991;118. 493-501.

21. Organización Mundial de la Salud. Decisiones de la Asamblea Mundial de la Salud. Cron OMS 1979;33:289-297.

22. Shann F, Hart K, Davis T. Acute lower respiratory tract infections: possible criteria for selection of patients for antibiotic therapy and hospital admissions. Bull WHO 1984;62: 749-753.

23. Shann F, Barker J, Poore P. Clinical signs that predict death in children with severe pneumonia. Pediatr Infect Dis 1989;8:12-17.

24. Véjar L, Navarrete P, Le-Cerf P. Educación a las madres; qué signos enseñar. Noticias sobre IRA 1995;32:6-8.

25. Chatkin J, Braga M, Crocópio M, Dalcolmo M. Estudio de óbitos infantis por pneumonia por meio de inquérito domiciliar. Pediatr 1992; $68: 3-4$.

Manuscrito recibido el 30 de agosto de 1996 y aceptado para publicación en versión revisada el 6 de junio de 1997. 
ABSTRACT This paper describes and examines the activities for the prevention and control of acute respiratory infections (ARI) carried out by a pediatric program in a government health service that provides care to a low-income population in Santiago, Chile. The study area has a population of just over one million people and is served by one general hospital, 16 primary care clinics, and eight rural health posts. Specific activities carried out by the ARI program include personnel training as well as control measures at the primary care level and hospitalization of children with severe ARI. In the first 5 years of the program (1990-1994), a reduction in annual mortality from pneumonia from 3.0 to 1.7 per 1000 was observed among children under the age of one year, which contributed to a decline in infant mortality from 13.5 to 8.9 deaths per 1000 live births. In 1994, program costs amounted to US\$ 224000 . Judging from the results obtained, the ARI prevention and control program has made a valuable con-

\section{Program for the prevention and control of childhood Santiago, Chile} tribution in the area of child health.

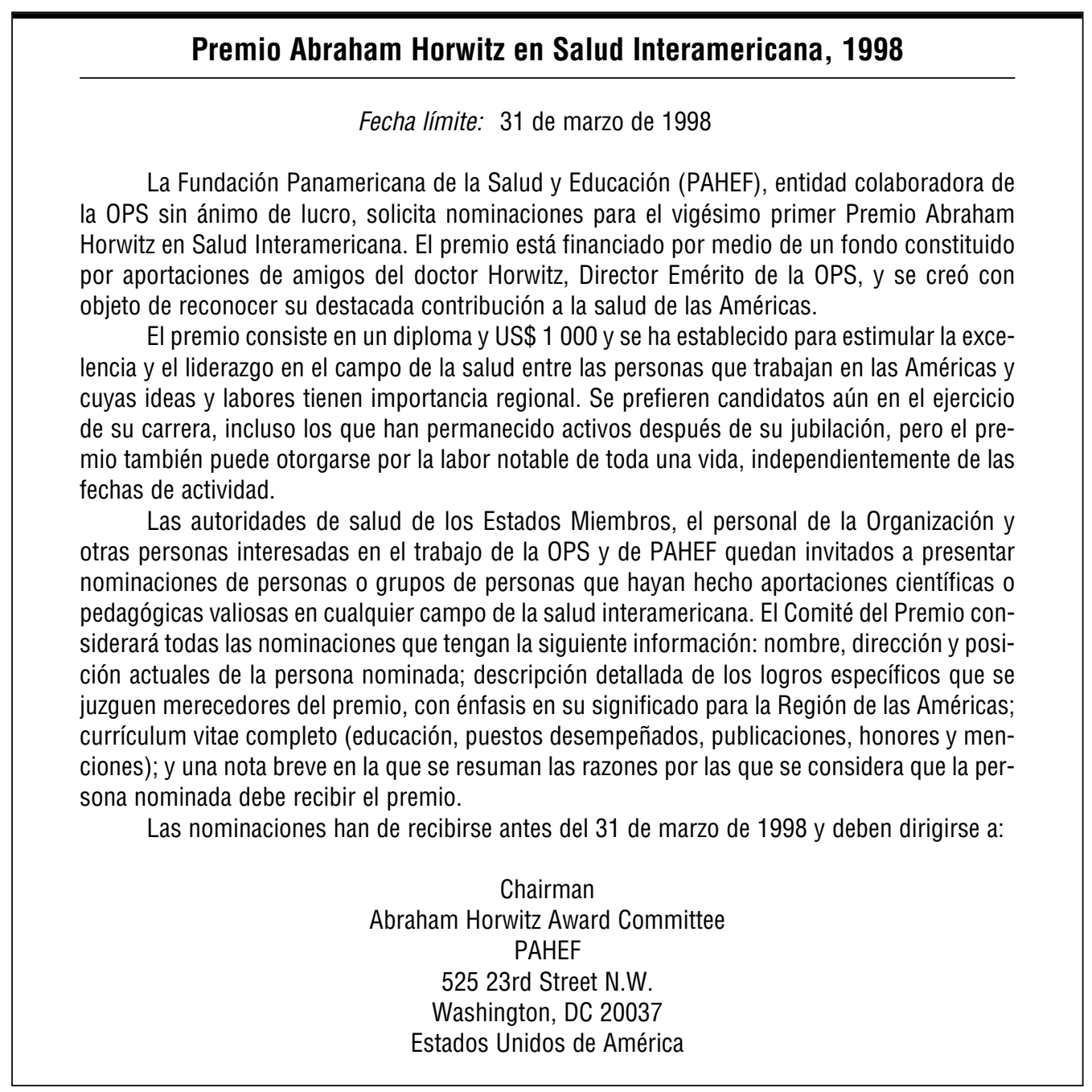

